\title{
DESIGN OF THE FERMILAB MAIN INJECTOR LAMBERTSON
}

\author{
D. E. Johnson, R. Baiod, D.J. Harding, P.S. Martin, M. May \\ Fermi National Accelerator Laboratory* \\ P.O. Box 500 \\ Batavia, Illinois 60510
}

\begin{abstract}
A common design has been adopted for a high field (1.1T) Lambertson magnet for use in the four injection/extraction channels of the Main Injector (MI) as well as the Tevatron injection channel [1]. To support the extraction/injection of $150 \mathrm{GeV} / \mathrm{c}$ protons and pbars, the field within the "field region" of the Lambertson should reach 1.1 Tesla with less than $0.3 \%$ variation in a 2 by 12 inch good field aperture. Utilization for the $8.9 \mathrm{GeV} / \mathrm{c}$ pbars and resonant extraction of $120 \mathrm{GeV} / \mathrm{c}$ protons place stringent aperture requirements on the magnet design. In addition, this magnet must simultaneously support circulating beam in the energy range of $8.9 \mathrm{GeV} / \mathrm{c}$ to $900 \mathrm{GeV} / \mathrm{c}$, through its "field free" region. At maximum excitation, the leakage field in this region must be kept to a minimum to avoid any deleterious effects on the circulating beam. The magnet design parameters which meet the aperture, magnetic, and structural requirements as well as the selection criteria of the steel are discussed.
\end{abstract}

\section{MAGNET REQUIREMENTS}

The extraction channels in the Main Injector utilize three vertically bending Lambertsons, with an integrated strength of $9 \mathrm{~T}-\mathrm{m}$, and a vertically bending c-magnet with an integrated strength of $3.5 \mathrm{~T}-\mathrm{m}$ to clear the downstream MI magnets. Four vertically bending Lambertsons, with an integrated strength of 12.1 T-m are required for injection into the Tevatron. Assuming a $1.1 \mathrm{~T}$ field for a symmetric Lambertson, imposed by tolerable leakage field, the magnetic length of the Lambetrson was set at 2.8 meters. This, in conjunction with the available free space in the MI lattice, set the flange to flange length at $3.054 \mathrm{~m}$.

The trajectory of $8.9 \mathrm{GeV} / \mathrm{c}$ pbars from the Antiproton Source through the de-energized set of Tevatron Lambertsons and into the MI define both the gap width (pole-to-pole dimension) and the minimum physical height of the gap (pole tip width). A minimum of $20 \%$ of the aperture is reserved for steering. The field free region must support circulating beam so the aperture defined by the height, width, and opening angle of this region should not be the limiting aperture. The maximum septa thickness is governed by the separation of the injected/extracted beam from the circulating beam. This separation is a function of the kicker or electrostatic septa strength. The physical aperture requirements for usage in both the MI and the Tevatron are listed in Table 1.

The good field height in the bend region, as listed in Table 2 , is determined by the maximum excursions of the extracted beam through the Lambertsons in both the MI extraction and the Tevatron injection channels. The field uniformity in this region

\footnotetext{
* Operated by the Universities Research Association under contract with the U. S. Department of Energy
}

should not contribute more than $1 \%$ to the emittance growth of the extracted beam.

Table I

Aperture Specifications

\begin{tabular}{|l|c|cl|}
\hline & MI & Tevatron & \\
\hline Gap width & 2 & 2 & inches \\
Gap height & \pm 5 & \pm 7 & inches \\
Field free region height & $\geq 2$ & $\approx 3$ & inches \\
Field free region width & 3.5 & 3.5 & inches \\
Opening angle & $\geq 90$ & $\geq 90$ & degrees \\
Septum Thickness & .157 & .157 & inches \\
Straightness (both planes) & 10 & 10 & mils \\
\hline
\end{tabular}

Besides the magnitude of the field and field quality in the bending region, the body leakage field into the field free region and the end field contributions, which impact the circulating beam, are specified. The specifications listed here set limits for the sum of the body and end field. Without any compensation, the end field contribution can be an order of magnitude larger than that due to the body leakage field. With careful attention, this can be reduced to the same order of magnitude as the leakage field. The current end field compensation scheme is discussed in a companion paper. [2]

Table II

Magnetic Specifications

\begin{tabular}{|l|c|cl|}
\hline & MI & Tevatron & \\
\hline Nominal field & 1.072 & 1.072 & Tesla \\
Good field height & \pm 4.6 & \pm 6 & inches \\
Field Uniformity & $<0.28$ & $<0.28$ & percent \\
Leakage Field & $<0.038$ & $<0.019$ & T-m \\
Leakage Gradient & $<.78$ & $<.336$ & Tesla-m/m \\
\hline
\end{tabular}

Due to the symmetry of the magnet, the predominant body leakage fields to contend with are the dipole, and skew quad. The magnitude of the allowed dipole field is specified to produce less than a $2 \mathrm{~mm}$ closed orbit distortion without downstream orbit compensation. From this the total allowed dipole field in the field free region is estimated and the results are listed in Table 2.

To set the upper limits on the skew quad field allowed in the field free region, the magnitude of the allowed tune shift (assuming a fully coupled ring) for the circulating beam in the field free region at maximum excitation has been specified to be less than 0.005 units. This criteria corresponds to 
$\int d B_{x} / d x d l<4 \pi \delta \nu(\beta \rho) / \beta$ in units of Tesla-m/m, again as a sum of the contributions from the body leakage field and the end field.

The project requires 16 Lambertsons plus spares, so a common design will reduce design effort, fabrication costs, and the quantity of required spares. Therefore, the design is based upon the requirements for usage in the Tevatron which automatically satisfies the Main Injector specifications.

\section{PHYSICAL DESIGN}

The requirement for a large aperture (both planes) in the bending region, led to the design of a Lambertson absent of an internal beam pipe. This is accomplished by using two sets of symmetric laminations, one set for the inner cores and one for the outer cores, as shown in Figure 1. The inner cores are assembled with a precision ground matched set of stainless steel space bars near each coil to define the gap width. The left/right symmetry of the inner cores assure an assembly which meets the straightness criteria in bend plane. This assembly is "skinned" with a 30 mil Type 321 stainless steel vacuum skin and terminated at each end by a single vacuum end plate. All vacuum welds are external. A set of 8 distributed 30 liter/sec ion pumps ( 4 on each side) are utilized to maintain a vacuum of $\approx 5 \times 10^{-9}$ torr for use in the Tevatron.

The coil is a 24-turn split saddle coil with the saddle extending laterally beyond the inner core to allow for the inner core end assembly to extend beyond the coil with out interference. The conductor dimensions and number of turns are determined by the desire to utilize an existing $200 \mathrm{Volt} / 2500 \mathrm{Amp}$ power supply while maintaining adequate copper volume and cooling.

The coil and inner core assembly are captured by a pair of symmetrical outer cores designed to provide straightness in the non-bend plane. The assembly is held together by a set of tie plates which produce stiff boxed beam structure.

Construction of previous Lambertsons at FNAL have required laborious straightening techniques of welding stripes on the outer core to meet the straightness criteria. This design deviates from the construction of previous Lambertsons at FNAL in that it is designed to meet the straightness criteria without the previously required straightening techniques.

\section{MAGNETIC DESIGN}

A 2D magnetic model was constructed to allow adjustment of basic geometrical parameters such as the septum thickness, septum radius, septum angle, and geometries of the field free region, inner core, outer core, gap height and width, pole face contour, and tiebars to aid in magnet design choices. The model additionally included the stainless steel skin around the inner cores and air gaps around the skin and tiebars. These parameters were adjusted to optimize the field uniformity in the gap, minimize the leakage field in the field free region, and trim the back leg.

\section{A. Field Region Design}

The uniformity in the central region of the gap is governed predominately by the location of the field free region. Its symmetric location provides excellent uniformity in this central re-

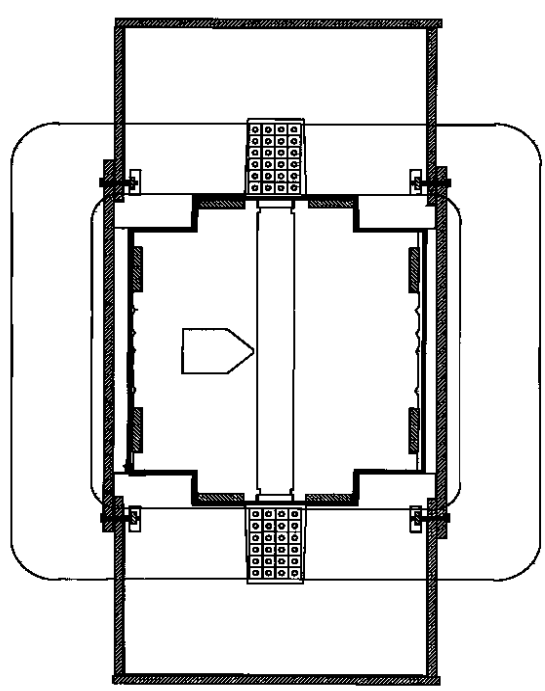

Figure 1. Cross section of Lambertson core showing inner and outer cores, tiebars, and coil configuration.

gion. The height of the "good field" region, defined by the uniformity specification, is governed by the coil and stainless steel spacer bar geometry and the shape of the pole tip near the coil. Without any pole tip shaping, the good field region extended to \pm 5 inches, about a gap width less than the physical height. Adjusting the width of the stainless spacer bar (i.e. its penetration into the iron of the inner cores) and adding a \pm 50 mil thick by 200 mil long pole tip shim increased the height of the good field region to \pm 6 inches, meeting the specification, as seen in the lower plot of Figure 2. However, the field falls off rapidly and increasing the the pole tip height from 7 to 8 inches linearly increases the height of the good field from 6 to 7 inches as shown in the upper plot of Figure 2.
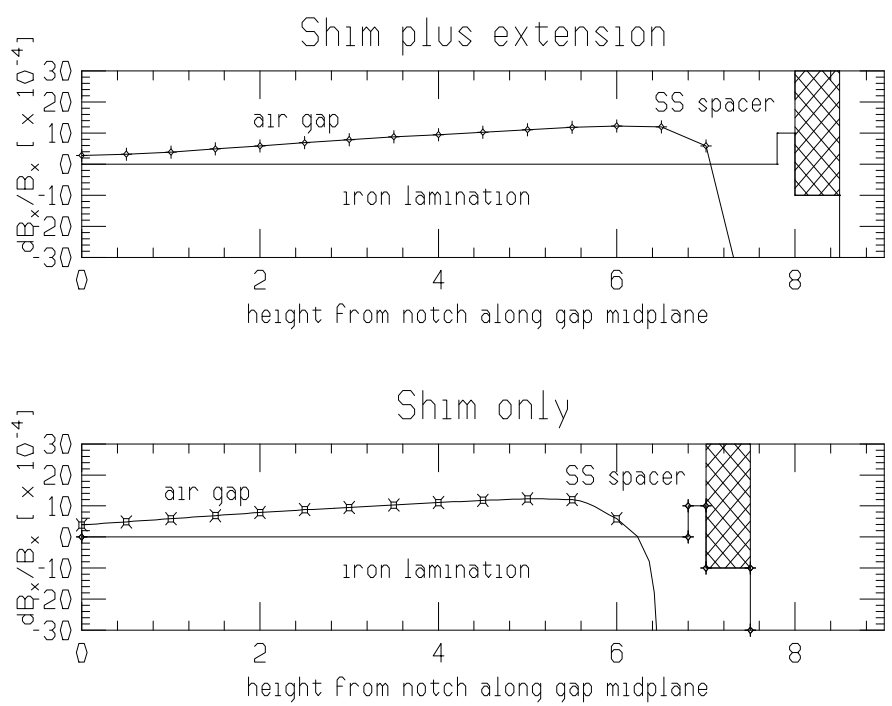

Figure 2. Calculated field uniformity in the bend region after shimming and extending the field region. 


\section{B. Field Free Region Design}

The leakage field from the iron into the field free region is governed by the continuity condition that $\mathrm{H}$ (parallel) must be continous across the iron/air interface. From this boundry condition, the flux density (in Gauss)in the field free region will just be $\mu_{0} H_{\text {steel }}$. To maintain control over the leakage field, a steel with a high permeability at the expected values of $\mathrm{H}$ in the iron near the cavity should be chosen. The choice of steel is discussed in the next section.

The selection of septum thickness, septum radius and opening angle are not only based upon the aperture requirements discussed earlier, but were selected to minimize the saturation in the iron near the septa, the magnitude of skew quad in the cavity, and the magnitude of the leakage flux density, respectively.

Figure 3 shows the magnitude of the field, in Gauss, on axis in the field free region as predicted by OPERA-2D. The small skew quad component is realized by a combination of reducing the opening angle from the 90 degree specificaion to 78 degrees and increasing the septum radius to 0.2 inches maintaining the vertical aperture near the notch. The magnitude of the field in the gap is 1.1 Tesla.

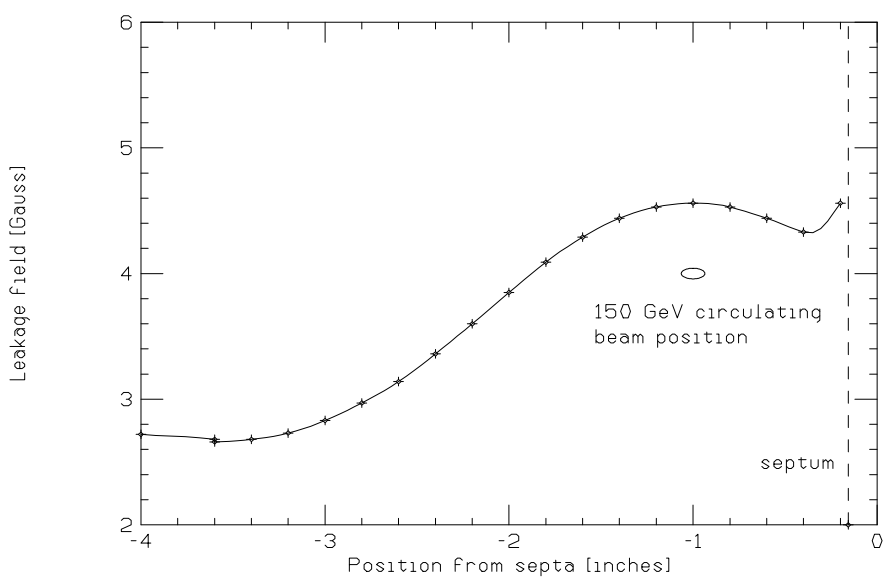

Figure 3. Leakage Field in the field free region at a bend field of 1.1T.

\section{Steel Selection}

The selection of steel is not only governed by technical specifications but by more realistic issues like market availability and cost. For example, cobolt/vanadium alloys such as supermendure offer a high permeability at the lower excitations, which would offer better shielding around the cavity or decrease the integrated magnet length. They are, however, cost prohibitive at $\approx \$ 30 / \mathrm{lb}$. as compared to $\approx \$ 0.50 / \mathrm{lb}$ for $\mathrm{Si}$ and low carbon steel. Therefore, we limited our selection of steel for the Lambertson magnet to either a silicon electrical steel or a low carbon steel. Figure 2 shows the measured hysteresis curves for 24 guage Epstein strip samples of a fully processed low carbon $(0.006 \%)$ steel and a M-47 grade Si steel. This shows the Si steel clearly having a higher permeability and a lower coercive force than the low carbon steel in the sheared condition (dashed line). Shearing and lamination punching increase stress within the steel which lowers its permeability. Both sets of samples were stress relief annealed at 750 degrees $\mathrm{C}$ for two hours. The permeability of both samples is shown as the solid lines. A more pronounced improvement from stress relief annealing is seen in the low carbon steel. For this project, the low carbon steel had acceptable parameters.
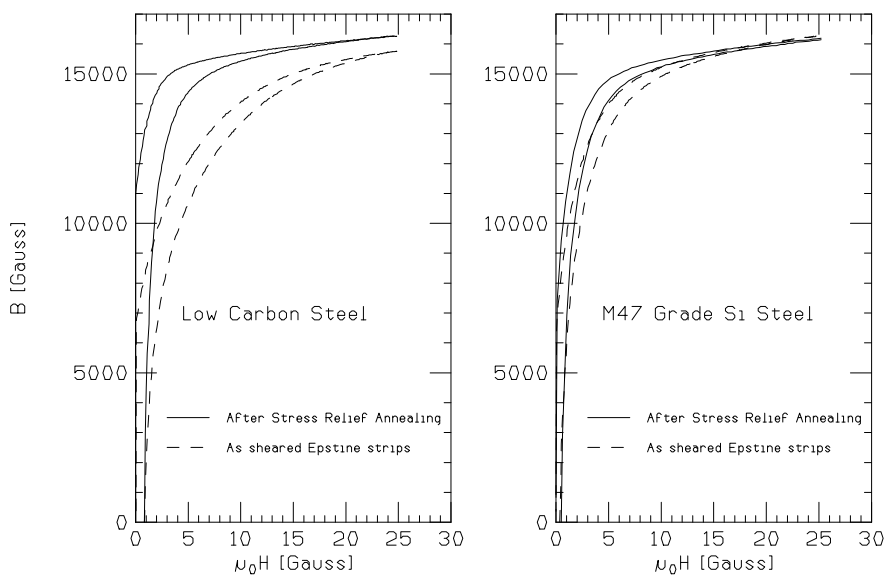

Figure 4. Hysteresis curves of low carbon and Si steel showing the improvement to each due to stress relief annealing.

\section{End Design}

A 30" prototype Lambertson has been constructed to aid in the design of the magnet end configuration. The ratio of magnetic length to physical (flange-flange) length must remain $\approx 92 \%$ due to space constraints in the Main Injector. The geometry of the prototype includes a 2 by 13 inch gap without any shimming. The field free region geometry used is listed in Table 1 .

\section{ACKNOWLEDGEMENTS}

The authors would like to thank Linda Alsip, John Carson, Nelson Chester, Gerry Davis, Fritz Lange, Arie Lipski, Gale Pewitt, Reid Rihel, Bill Robotham, Kay Weber, John Zweibohmer, for their contributions to this design.

\section{References}

[1] The Fermilab Main Injector Technical Design Handbook, August 1994.

[2] J.-F. Ostiguy and D.E. Johnson, "3D End Effects in Iron Septum Magnets", these proceedings. 